\title{
ㄷำ1
}

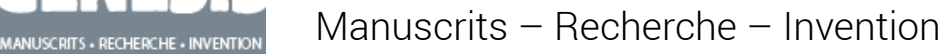

38 | 2014

Traduire

\section{Les deux corps du texte}

Fabienne Durand-Bogaert

\section{OpenEdition}

\section{Journals}

Édition électronique

URL : http://journals.openedition.org/genesis/1284

DOI : 10.4000/genesis. 1284

ISSN : 2268-1590

\section{Éditeur :}

Presses universitaires de Paris Sorbonne (PUPS), Société internationale de génétique artistique littéraire et scientifique (SIGALES)

\section{Édition imprimée}

Date de publication : 15 avril 2014

Pagination : 11-33

ISBN : 9782840509370

ISSN : 1167-5101

\section{Référence électronique}

Fabienne Durand-Bogaert, «Les deux corps du texte », Genesis [En ligne], 38 | 2014, mis en ligne le 27 juin 2016, consulté le 11 juin 2020. URL : http://journals.openedition.org/genesis/1284 ; DOI : https:// doi.org/10.4000/genesis.1284 


\title{
Les deux corps du texte
}

\author{
Fabienne Durand-Bogaert
}

$\mathbf{P}$ armi les projets que le Collège international de philosophie avait inscrits à son programme, lors de sa fondation, il y a trente ans, figurait la réflexion sur le traduire, insérée entre ces deux pans de l'activité de l'esprit que sont le «lire » et l'« interpréter » (fig. 1). C'est dans ce cadre qu'Antoine Berman dispensa, entre 1984 et 1989, plusieurs séminaires dont l'impact fut décisif pour l'édification d'une pensée de la traduction novatrice et féconde. Loin des approximations qui ont longtemps défiguré l'acte de traduction, le réduisant à la négociation d'un compromis entre les logiques linguistiques, textuelles, culturelles - de deux langues que leur autonomie respective rendait difficilement conciliables, Antoine Berman s'est attaché à dégager la spécificité du traduire en fonction de trois grandes visées méthodologiques : l'analyse des caractéristiques stylistiques propres d'une traduction, la reconstitution du contexte ou de l'« horizon » sur fond duquel cette traduction a surgi, la mise en évidence de la position du traducteur à l'égard de son travail. Ce faisant, Berman a posé les bases d'une critique des traductions qui, bien qu'elle ne se soit jamais réclamée de l'approche génétique1, n'en a pas moins rodé certains outils qui se révèlent aujourd'hui précieux pour quiconque cherche à cerner les processus par lesquels « une ouvre passe d'une langue à une autre ou ne cesse de re-passer, au fil des re-traductions, sur les rives de notre langue maternelle en se métamorphosant et en métamorphosant les langues de départ et d'arrivée 2 ».

1. La démarche relèverait plutôt d'une archéologie des traductions au sens foucaldien du terme, comme il est précisé dans l'édition posthume de l'ouvrage d'Antoine Berman, Jacques Amyot, traducteur français : essai sur les origines de la traduction en France (Paris, Belin, coll. «L'extrême contemporain », 2012) : « Pour comprendre notre tradition de traduction, il faut [...] se défaire d'un certain nombre de préjugés. Il faut, ensuite, s'interroger sur la persistante occultation du rôle fondateur de la traduction en France. Il faut enfin, et surtout, remonter à l'origine de cette tradition, par un mouvement proprement archéologique » (p. 19).

2. Antoine Berman, «Critique, commentaire et traduction », Po\&sie, n 37, Paris, Belin, 1986, p. 102. C'est précisément ce moment du passage, et ses modalités, qui figurent, selon Berman, l'impensé de la critique, qui «n'en parle guère ou, lorsqu'elle en parle, [le fait] soit avec parcimonie, soit avec dédain, soit avec distance » (ibid.). 


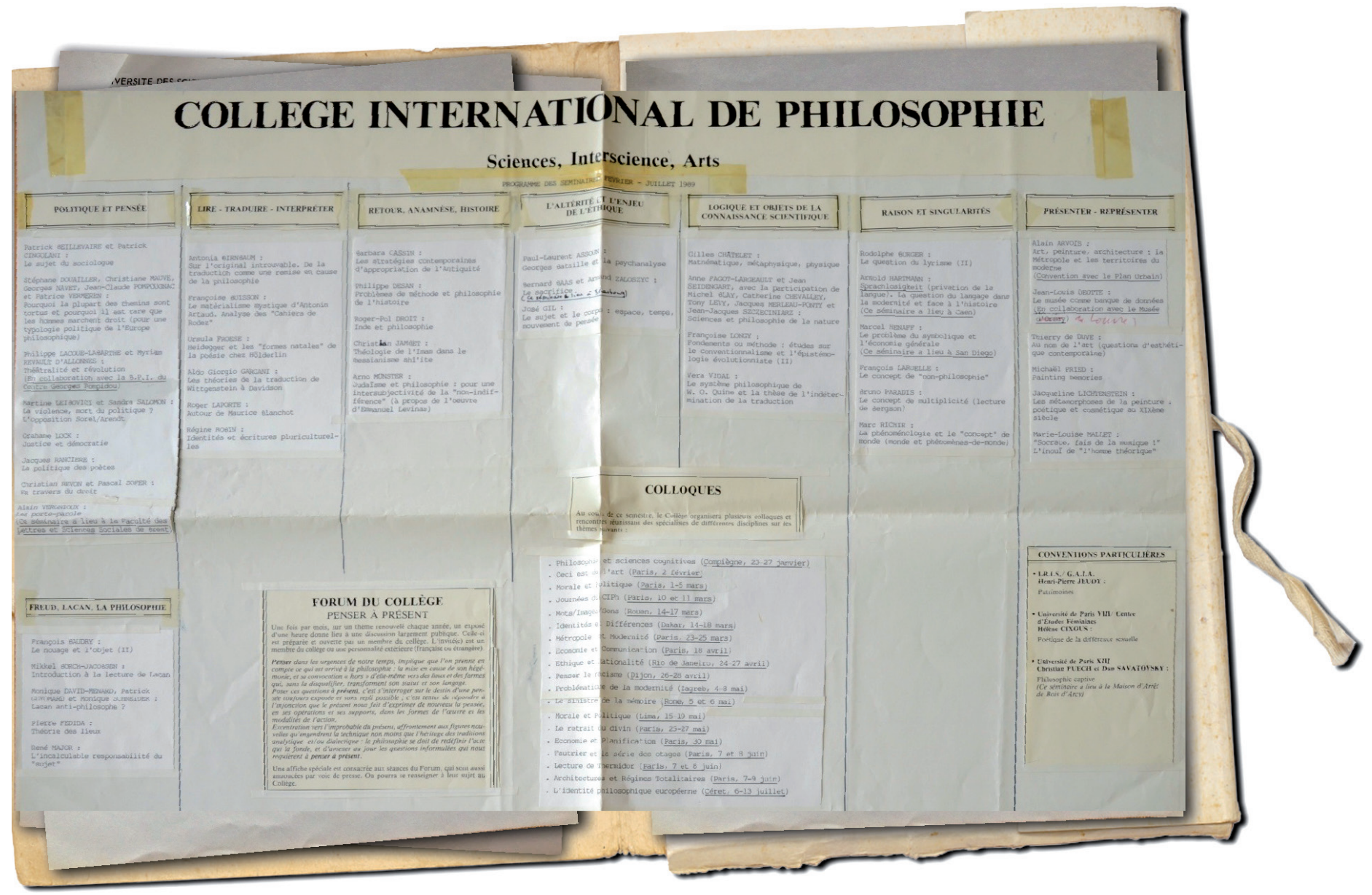

Fig. 1 : Programme du Collège international de philosophie, 1983

Aussi énigmatique que le processus de la création littéraire ou artistique, la genèse du traduire n'a guère encore été étudiée. Les raisons de cette lacune sont, pour une bonne part, imputables à deux facteurs étroitement liés, historiquement et ontologiquement : la secondarité de la traduction par rapport à ce que l'on appelle couramment l'« original » (terme que je commenterai plus loin), et la très ancienne réticence à accorder au traducteur le statut d'auteur. On comprendra mieux quelle configuration particulière se présente au généticien de la traduction si l'on précise d'abord les principales implications de cette situation dont l'intériorisation par les traducteurs ne favorise guère la disponibilité du matériau génétique.

\section{La secondarité de la traduction}

Qu'il surgisse ou non « organiquement », comme le pensait Walter Benjamin, de l'original, le texte d'une traduction est toujours un texte second renvoyant à un texte premier et, de ce fait, le confirmant dans son statut d'« original ». À cet égard, on pourrait même dire que la traduction «fait » l'original, du moins dans une perspective génétique. Venant après coup, la traduction n'a pas l'autonomie de l'original : elle existe dans ce qu'on appellera, selon le degré de dépendance qu'on lui prête, sa suite, son sillage ; elle en est l'une des émanations ou dérivations possibles (chacun de ces termes, on le pressent, 
propose à la pensée une bifurcation particulière qu'il serait intéressant de suivre jusqu'au bout). La secondarité temporelle de la traduction est donc une donnée même de la forme traduction. Le problème, au moins depuis la fin de la Renaissance, est que cette secondarité temporelle a été source de bien des confusions qui, à terme, ont abouti à une minoration du statut de la traduction. De «postérieure » à l'original (Benjamin disait «plus tardive »), la traduction lui est devenue « subordonnée » au sens infériorisant du terme. D'un point de vue sémantique, l'ancillarisation de la traduction a sans doute été facilitée par l'abondance des métaphores, qui ont longtemps constitué le principal discours tentant d'appréhender comme on dit d'un suspect - l'objet traduction : on trouvera, de l'Antiquité à aujourd'hui, nombre d'écrits, y compris des traducteurs eux-mêmes, dans lesquels la traduction est régulièrement dite «copie », « imitation » (fût-ce « la plus vraie », selon Jacques Peletier du Mans, au Xvie siècle), « envers d'une tapisserie » (Plutarque), mais aussi « vetement », «parure » qu'on endosse pour paraître sous une autre forme, "déguisement », voire « travestissement», etc. Quant au traducteur, l'adage est trop connu pour qu'on rappelle ici ce que fait peser sur lui la double contrainte consistant à « servir deux maîtres ».

En posant qu'il ne peut y avoir de passage qu'entre éléments distincts, la linguistique a introduit un peu d'ordre et de science dans le discours sur la traduction ${ }^{3}$. Aux approximations métaphoriques, elle a opposé des outils conceptuels tels que «langue de départ » et « langue d'arrivée », " source » et " cible », liées l'une à l'autre par une opération de décodage. La source a encodé un message dans une langue et une forme particulières qu'il faut d'abord décoder si l'on veut le transporter, encodé dans une autre langue et dans une forme ressemblante, jusqu'à la cible. Que le trajet ait des allures de jeu de piste n'a jamais vraiment retenu l'attention des linguistes, du moins en dehors des schémas de la communication où le «brouillage » a été amplement commenté. Ainsi, dans son souci légitime de pallier le manque d'une définition opératoire de la traduction, la linguistique s'est-elle concentrée davantage sur la polarité langue source/langue cible, point de départ/point d'arrivée que sur le moment du passage - les événements qui le jalonnent, les traces qu'il laisse derrière lui. Il n'est pas impossible qu'en négligeant le moment du passage, la linguistique ait contribué à entretenir l'illusion déjà fortement enracinée selon laquelle la vertu principale d'une traduction est sa transparence ${ }^{4}$.

3. Jean-René Ladmiral rappelle le rôle important qu'a joué la linguistique dans la réflexion sur la traduction : « Pour des raisons qui tiennent à l'histoire des sciences humaines, c'est dans le cadre de la linguistique qu'il a été question de la traduction - quand il en a été question, c'est-à-dire très peu. Il y avait là une certaine logique dans la mesure où la linguistique fournit une méthodologie et une terminologie qui permettent d'étiqueter les réalités évidemment langagières avec lesquelles la traduction a affaire et de les conceptualiser. Il reste que ce n'est que par une approximation provisoire qu'on avait cru devoir faire de la traductologie un chapitre, une sous-discipline de la linguistique ; outre-Rhin, on tend même à l'identifier à la Linguistique Appliquée (l'anglicisme de la double majuscule étant censé marquer ici la cohérence d'une spécialité à part entière) » («Préface à la seconde édition » de Traduire : théorèmes pour la traduction, Paris, Gallimard, coll. « Tel », 1994, p. VII-VIII).

4. On songe, par exemple, à la théorie des « verres transparents » de Georges Mounin (Les Belles Infidèles, Paris, Cahiers du Sud, 1955). Selon Benjamin, la vraie traduction est non pas transparente, mais translucide [durchscheinend], elle n'occulte pas l'original, ne se met pas devant sa lumière [ «steht ihm nicht im Licht »]. Sur la différence entre transparent et translucide, voir A. Berman, L'Âge de la traduction, Paris, Presses universitaires de Vincennes, 2008, p. 168. 
Les généticiens, avec juste raison, se méfient de certains termes. «Original », par exemple, à moins d'être accolé à un autre mot tel «édition » et d'en prendre une signification bien précise, est un terme dont l'emploi est problématique, étant donné les connotations qu'il suscite. Voici ce que l'on peut lire à l'entrée « Original » du Dictionnaire historique de la langue française :

Original est employé depuis l'ancien français comme nom (1269) pour désigner le manuscrit primitif d'un écrit, à la fois par opposition à copie, traduction et reproduction, selon le contexte. Dans une conception de l'art comme imitation du réel (mimesis), il a désigné, aux xvie et XVII ${ }^{e}$ siècles, un objet ayant servi ou susceptible de servir de modèle à un peintre, à un sculpteur (1563), emploi supplanté par modèle. Par suite, il a pris le sens qualitatif de « modèle d'excellence, digne d'être imité », en parlant de personnes ou de choses. Ainsi Furetière cite-t-il Homère et Virgile comme des originaux et Molière comme original en son genre, la comédie.

Cette définition met en évidence le passage qui s'est opéré de l'idée d' «original », immédiatement associée à celle d'unicité, à celle de modèle susceptible d'engendrer des dérivés, puis de "modèle d'excellence », nécessairement intouchable et inégalable. Le mot « original », cependant, ne semble pas avoir embarrassé outre mesure les traductologues, qui continuent d'en accepter l'emploi somme toute banalisé. Or, pour la traduction, cet emploi n'est pas sans conséquences : postuler le caractère fondamentalement « original » d'un texte, c'est déjà l'isoler en lui-même comme artefact unique digne d'être préservé, et donc instaurer un climat de méfiance à l'égard de toute opération qui, le " touchant », risque de menacer son intégrité. Pour une bonne part, c'est de cette sacralisation de l'original que procèdent toutes les idées négatives - ou faut-il dire « reçues », car ce sont souvent les mêmes - qui sont encore trop souvent associées à la traduction. Sans vouloir prétendre en dresser une liste exhaustive, on peut cependant énumérer les plus courantes. L'idée, par exemple, que la défaillance est comme consubstantielle à la traduction, soit que la traduction manque l'original (celui-ci résiste, affirme son unicité, sort encore grandi de l'affrontement), soit qu'elle l'atteigne, mais alors cela ne saurait être que par endroits, à preuve le fait que le lecteur d'une traduction ne lui accorde jamais toute la confiance qu'il accorde à l'original. D'où la réputation d' « intraduisibilité » de certaines œuvres - au mépris, du reste, des traductions existantes - qui, confinées dans leur unicité, ne se laissent soi-disant pas transporter. La sacralisation de l'original et son corrélat, la méfiance à l'égard des traductions, ne sont pas non plus sans incidence sur l'abord critique qu'ont suscité les traductions. En fait de «critique » des traductions, il s'est longtemps agi de jugements à l'emporte-pièce qui, loin de toute analyse rigoureuse, semblaient prendre un malin plaisir à mettre en évidence la richesse et la subtilité de la texture de l'original pour mieux déplorer l'insuffisance de la traduction'5. Ce n'est qu'à partir des travaux de l'École de Tel Aviv,

5. Contrastant « recensions critiques » des traductions, qui existent depuis l'âge classique, mais ont toujours été fortement teintées de jugement moral, et la critique des traductions telle qu'il en « dessine [...] les contours », Berman écrit : « Mais si critique veut dire analyse rigoureuse d'une traduction, de ses traits fondamentaux, du projet qui lui a donné naissance, de l'horizon dans lequel elle a surgi, de la position du traducteur; si critique veut dire, fondamentalement, dégagement de la vérité d'une traduction, alors il faut dire que la critique des traductions commence à peine à exister » (Pour une critique des traductions : John Donne, Paris, Gallimard, coll. « Bibliothèque des idées », 1995, p. 13-14). 
dans les années soixante-dix, qu'a pu s'amorcer une critique des traductions visant à systématiser l'analyse des textes traduits sur la base d'échantillons imprimés6.

On peut aisément déduire, de ce qui précède, combien l'auctoritas du traducteur est fragile, quand elle ne lui est pas déniée. La confusion entre les deux régimes - temporel et ontologique - de secondarité qui affecte la traduction rejaillit sur la figure du traducteur, au fil de l'histoire, pour le vouer à l'effacement. George Steiner a parfaitement résumé la nature du problème, qui écrit dans Après Babel :

La traduction ne constitue pas un élément de premier plan de l'histoire et de la théorie de la littérature. Elle y figure, au plus, de façon marginale. La seule exception est l'étude de la transmission et de l'interprétation du canon biblique. Mais il s'agit là d'un domaine bien particulier dans lequel le problème de la traduction n'est rien d'autre qu'un aspect du système plus vaste de l'exégèse. Il n'existe pas de traité de la traduction comparable, par le poids et l'étendue, à celui de Longin sur le sublime ou à la Poétique d'Aristote. Il n'y a que peu de temps, depuis la création en fait de l'International Federation of Translators à Paris en 1953, que les traducteurs s'affirment en tant que corps professionnel et réclament un statut corporatif. Jusque-là, la description que donne Valery Larbaud du traducteur en train de mendier sous le porche de l'église contenait beaucoup de vrai. «Le traducteur est méconnu; il est assis à la dernière place ; il ne vit pour ainsi dire que d'aumônes. » À ce jour encore, les compensations financières qui accompagnent la traduction sont la plupart du temps dérisoires en regard de la difficulté et de l'importance du travail impliqué. Bien que l'Index translationum publié chaque année sous les auspices de l'Unesco reflète une augmentation saisissante du nombre et de la qualité des ouvrages traduits, bien que la traduction soit vraisemblablement, à elle seule, l'arme la plus évidente dans la bataille pour l'acquisition de la science et de la conscience dans les pays en voie de développement, le traducteur n'a souvent pas plus d'épaisseur qu'un fantôme. C'est tout juste si on le remarque au dos de la page de garde. Qui ira relever son nom ou éprouver de la gratitude pour le travail qu'il a accompli ? Il en a toujours été plus ou moins ainsi7.

Steiner rappelle ensuite les noms oubliés de divers traducteurs, dénonce la critique qui, en Angleterre, s'émerveille de l'influence colossale qu'a eue Werther mais passe sous silence le rôle essentiel des traducteurs en tant qu'agents du rayonnement de l'œuvre, et conclut sur une note à peine plus optimiste : « Il n'y a pas longtemps que le traducteur [...] a commencé à se dégager d'un arrière-plan de grisaille et de servitude. Et encore tient-il souvent lieu de cible : on découvre qui a donné accès à Proust et Dostoïevski quand on s'aperçoit que le travail demande à être refait. »

Dans le fond et pour l'essentiel, la situation que décrivait George Steiner en 1976 n'a guère changé. Il suffit, pour s'en convaincre, de lire La Condition du traducteur, l'ample

6. Ce n'est pas ici le lieu de décrire ces travaux, mais il convient de rappeler qu'ils constituent une étape décisive de la traductologie telle qu'elle s'est ensuite développée au Canada et en Belgique. C'est la base édifiée par l'École de Tel Aviv que Berman a élargie en montrant que la critique des traductions ne pouvait s'en tenir à l'approche linguistique et sémiotique de son objet : qu'elle avait besoin de la philosophie pour conceptualiser ses outils; de l'histoire, pour situer une traduction donnée dans le contexte culturel où elle est apparue ; de la sociologie, pour éclairer la figure du traducteur, et de la psychanalyse pour comprendre quels ressorts animent ce que Berman nommait la «pulsion du traduire».

7. George Steiner, Après Babel. Une poétique du dire et de la traduction, trad. L. Lotringer et P.-E. Dauzat, Paris, Albin Michel, 1998, p. 369-371. 


\section{Quelle archive pour la traduction?}

étude réalisée, entre 2009 et 2011, par Pierre Assouline à la demande du Centre national des lettres. Le titre est d'ailleurs en lui-même éloquent, si l'on songe que le mot « condition » est tombé en déshérence, dans la France du $\mathrm{XxI}^{\mathrm{e}}$ siècle, au point de ne même plus s'appliquer à ces « minorités » que furent jadis les femmes ou les travailleurs immigrés...

Secondarité du texte traduit, auctoritas fragile du traducteur (rendue plus fragile encore du fait qu'il n'est pas le seul à réviser sa copie), marginalité de la traduction au sein de l'espace littéraire et culturel, telles sont les données avec lesquelles le généticien de la traduction doit composer. Nul doute qu'elles ne compliquent quelque peu sa tâche, ne serait-ce que parce que l'archive des traducteurs n'a jamais suscité, de la part des bibliothèques, des éditeurs, des ayants droit, voire des intéressés eux-mêmes, le même souci de préservation que l'archive des écrivains. Quelques fonds ont commencé à se constituer dans certaines institutions, notamment à la Bibliothèque nationale de France et à l'Institut Mémoires de l'Édition contemporaine (IMEC), mais une réelle politique d'identification et de conservation de l'archive relative aux traductions n'a encore pas vu le jour en France, où la situation de la traduction et le statut des traducteurs sont pourtant plus favorables que dans bien d'autres pays d'Europe ou du monde. L'archive doit donc souvent être débusquée et se révèle volontiers parcellaire, notamment en ce qui concerne les différents états des manuscrits ou tapuscrits - pour ne rien dire des cas, de plus en plus fréquents à l'époque contemporaine, où la seule forme matérielle que prend le travail du traducteur est celle que lui donne l'éditeur en publiant la traduction ${ }^{8}$. Les textes traduits étant en majorité des œuvres de commande, le «produit fini »a, pour la plupart des éditeurs et des traducteurs eux-mêmes, plus de valeur que les processus par lesquels il prend forme. Dans ces conditions, il n'est guère surprenant qu'un éditeur, par exemple, ne s'attarde pas à conserver ni à gérer les traces de ce qui précède et entoure la traduction. Ce sont ainsi des pans entiers d'une très grande valeur épistémologique pour le chercheur qui disparaissent. J'en ai moi-même fait le constat lors du travail que j'ai mené sur les traductions françaises de Henry James ${ }^{9}$ : de Louise Servicen, l'une des grandes traductrices attachées aux éditions Albin Michel dans les années cinquante, ne subsistaient plus que trois ou quatre lettres d'une correspondance avec le directeur de la maison, suffisantes pour entrevoir l'intérêt qu'il y aurait eu à disposer d'un fonds plus fourni, mais trop maigres pour servir d'appui à l'objectif que je m'étais fixé : reconstituer la genèse de la traduction du The Last of the Valerii ${ }^{10}$. Or la maison d'édition était mon dernier recours, après que j'eus vainement sondé les bibliothèques et recherché

8. J'ai moi-même pu constater, ces dernières années, le caractère de plus en plus immatériel de mon travail de traductrice. Nombre de textes à traduire me sont envoyés par courrier électronique ; une fois le texte traduit (je travaille directement sur l'ordinateur), je le relis sur écran et l'envoie à l'éditeur par la même voie ; ce sera aussi celle qu'il utilisera pour me faire part, en retour, de ses éventuels commentaires et m'envoyer les «épreuves » (sous forme de PDF) de ma traduction. Je n'ai donc affaire, avant la publication de ma traduction, qu'à un seul texte imprimé : le texte à traduire, que j'imprime moi-même, pour le traduire, et qu'il m'arrive d'annoter.

9. Pour ma thèse de doctorat: De la séparation: Henry James du point de vue de ses traductions en langue française, EHESS, 1999.

10. Nouvelle de 1874, qui semble avoir été inspirée à Henry James par la lecture de La Vénus d'Ille, de Prosper Mérimée. Une première traduction française, d'Auguste Monod, fut publiée dans La Revue des Deux Mondes en 1875. 
les descendants de Servicen. Que je ne trouve, dans la chemise portant son nom, ni notes préparatoires, ni trace d'un quelconque état manuscrit ou tapuscrit, ni même une simple coupure de presse évoquant la publication de la traduction fut non seulement une source de déception, mais aussi d'inquiétude quant à la possibilité même de mener une recherche génétique sur les textes traduits.

Pouvons-nous espérer que la moisson soit plus abondante si nous nous adressons aux traducteurs qui sont en activité aujourd'hui ? Rien n'est moins sûr, à en juger par l'enquête que Jean-Louis Lebrave et moi-même avons menée auprès d'un échantillon de traducteurs qui étaient associés, individuellement ou collectivement, à une traduction d'importance11. Nous leur avons demandé ce qu'ils étaient prêts à montrer à un regard étranger. Les réponses furent instructives : en ce qui concerne les entreprises collectives - retraduction de la Bible aux éditions Bayard, séminaires de traduction-poésie de Royaumont, directions de collection de littérature étrangère -, rien ne s'opposait, dans l'esprit des personnes interrogées, à ce que l'archive générée soit rendue accessible à qui pouvait s'y intéresser (les chercheurs, en premier lieu). Mais toutes sortes de réticences s'exprimèrent en ce qui concerne les traductions que ces mêmes traducteurs réalisaient à titre individuel. Certains affirmèrent que la seule archive d'un traducteur est sa bibliothèque, dont il ne peut se séparer, du moins de son vivant. D'autres déclarèrent ne conserver aucun document relatif à leur travail hormis la correspondance qu'ils échangent avec l'auteur, trop personnelle pour être livrée au regard d'autrui. D'autres encore se dirent soulagés de pouvoir détruire, une fois la traduction publiée, les traces de ce qui l'avait accompagnée : carnets de notes contenant des informations recueillies en bibliothèque, dictionnaires personnels qu'ils s'étaient constitués d'après l'idiolecte d'un écrivain ou le lexique d'un domaine spécifique, tirages papier témoignant de différentes phases du travail, etc. (Poussés dans leurs retranchements, ils nous confièrent que c'étaient là autant de rappels de la souffrance attachée à une tâche ressentie très souvent comme «ingrate », toujours comme « solitaire ».) Beaucoup invoquèrent le fait qu'une traduction est une œuvre de commande moins «personnelle » qu'un livre qu'on écrit pour expliquer le peu de cas qu'ils faisaient du processus et du matériau qu'elle génère. Enfin, l'argument de l'urgence fut plus d'une fois invoqué : sommés de venir à bout de leur traduction dans des délais souvent très brefs, les traducteurs n'ont que le temps du trajet, pas celui du retour réflexif sur leurs traces.

Loin de nous décourager, ces témoignages nous commandent seulement d'être vigilants et convaincants : il appartient aux chercheurs, me semble-t-il, d'une part d'inciter les traducteurs à préserver leurs documents de travail ; d'autre part de les encourager à accepter de se montrer dans leurs états préparatoires, leurs brouillons, leurs repentirs - ce qu'ils feraient sans doute plus volontiers si la traduction en tant que forme bénéficiait d'une aura aussi positive que celle de l'écriture. Mais la réciproque vaut aussi : nul doute que l'approche génétique ne puisse contribuer à ébranler certaines conceptions figées de

11. Furent interrogés, entre autres, Claude Esteban et Rémy Hourcade (poètes-traducteurs de Royaumont), Marianne Alphant (pour sa participation à la traduction de la Bible éditée chez Bayard en 2001), René de Ceccatty (traducteur du japonais et directeur de collection au Seuil), Jean-Yves Masson (traducteur et directeur de la collection « Der Doppelgänger » chez Verdier), lors de deux journées de travail qui se tinrent à l'IMEC en juin 2001. 
la traduction et de la figure du traducteur. Pour Antoine Berman, « aller au traducteur » représentait un « tournant méthodologique [...] essentiel » qu'il appelait de ses vœux :

[...] il devient de plus en plus impensable que le traducteur reste ce parfait inconnu qu'il est encore la plupart du temps. Il nous importe de savoir s'il est français ou étranger, s'il n'est « que » traducteur ou s'il exerce une autre profession significative, comme celle d'enseignant (cas d'une très importante portion de traducteurs littéraires en France) ; nous voulons savoir s'il est aussi auteur et a produit des œuvres ; de quelle(s) langue(s) il traduit, quel(s) rapport(s) il entretient avec elle(s); s'il est bilingue, et de quelle sorte ; quels genres d'œuvres il traduit usuellement, et quelles autres œuvres il a traduites; s'il est polytraducteur (cas le plus fréquent) ou monotraducteur (comme Claire Cayron ${ }^{12}$ ) ; nous voulons savoir quels sont, donc, ses domaines langagiers et littéraires ; nous voulons savoir s'il a fait œuvre de traduction au sens indiqué plus haut et quelles sont ses traductions centrales ; s’il a écrit des articles, études, thèses, ouvrages sur les œuvres qu'il a traduites; et enfin, s'il a écrit sur sa pratique de traducteur, sur les principes qui la guident, sur ses traductions et la traduction en général ${ }^{13}$.

À quoi j'ajouterai qu' « aller au traducteur » constitue aussi une prise de position idéologique à l'encontre des théories qui postulent sa nécessaire "transparence », voire son « invisibilité ». Entreprendre une critique génétique des traductions, c'est donc non seulement chercher à comprendre quelle est la physionomie du texte traduit, mais aussi qui est ou a été le traducteur - quel type de rapports il a noué à sa pratique, et par-delà à l'écriture, à la poétique de son temps, au langage. En somme, quel être-au-monde s'est trouvé mis en jeu, dont le texte traduit nous restitue nécessairement quelque chose. On conçoit aisément qu'une «traduction d'écrivain »-Les Vagues par Marguerite Yourcenar, par exemple, ou L'Énéide par Pierre Klossowski - puisse porter les traces d'une exploration des possibilités de la langue qu'affirme parallèlement une œuvre de fiction du même auteur. Mais on prête moins d'attention à cette hypothèse dès lors qu'un traducteur mène la dite exploration dans l'espace même de ses traductions, voire lui donne des prolongements dans d'autres écrits. Avant de détailler les opérations génétiques qu'il est possible de mener sur les textes eux-mêmes (texte source et texte traduit), j'éclairerai d'un exemple - celui de Jean Pavans - la notion de projet de traduction telle qu'elle se profile à travers un matériau que l'on peut assimiler au paratexte.

\section{Jean Pavans ou la volonté d'intégrale}

L'entreprise de Jean Pavans occupe une place singulière dans le paysage des traductions françaises de Henry James. Né en 1949 à Tunis, Pavans est venu à la traduction dans les années quatre-vingt, alors que le désir de donner son temps à l'écriture, bientôt suivi de la publication de trois ouvrages ${ }^{14}$, le poussait à renoncer à une première carrière de mathématicien spécialiste des questions de logique. Sa rencontre avec l'œuvre de James est, à

12. Traductrice de Miguel Torga (voir infra, note 16).

13. Pour une critique des traductions : John Donne, op. cit., p. 73-74.

14. Publiés aux éditions de la Différence, en 1980 : Lazare définitif, récit ; Sauna, pastiches ; et Ruptures d'innocences, roman. Depuis lors, aux milliers de pages jamesiennes traduites, Jean Pavans a ajouté plusieurs essais, dont Heures jamesiennes (éd. de la Différence, 2008) et de nombreuses adaptations théâtrales, radiophoniques et même opératiques (l'opéra de La Bête dans la jungle fut créé en mai 2011, sur un livret de Pavans). 
l'origine, fortuite. C'est un ami, lecteur des récits qu'avait publiés Pavans, qui lui a conseillé d'explorer l'œuvre jamesienne, frappé qu'il était par nombre d'affinités entre l'écriture et l'univers des deux auteurs. La découverte, à la Bibliothèque publique d'information du Centre Pompidou, de plusieurs récits de James, dont The Sacred Fount (1901), eut tôt fait de convaincre Jean Pavans qu'il y avait là matière à une longue fréquentation 15 . L'intuition était bonne et ne s'est jamais démentie, dotant Pavans de l'énergie nécessaire à la poursuite d'un immense projet : la traduction de l'intégrale des quelque cent vingt nouvelles publiées par James entre 1864 et 1910. Entamé en 1980, le travail s'acheva en 2009 avec la parution, aux éditions de la Différence, d'un quatrième et dernier volume.

Ces données - sentiment d'une profonde et essentielle affinité avec l'écrivain d'où découle l'exigence qu'à une unité de textes corresponde une unité de voix - sont le fond sur lequel l'entreprise de Jean Pavans se distingue des traductions antérieures de Henry James. À la différence des traductions relativement éparses et isolées qui jalonnent la translation française de l'écrivain, les traductions de Jean Pavans forment un tout, s'inscrivent dans un désir d'œuvre que le temps a constamment aiguisé.

D'une particulière importance est ici le projet général du traducteur, dans la mesure où Jean Pavans ne s'est pas laissé guider par une quelconque demande de l'édition française mais a su, par la cohérence de ses choix, imposer une entreprise dont il avait l'entière initiative. Il l'a fait, qui plus est, dans un pays où il n'est pas habituel de voir un traducteur s'attacher à l'œuvre - en l'espèce, monumentale - d'un même auteur ${ }^{16}$. D'entrée de jeu, cette posture suggère un questionnement quant à la nature des liens qui unissent fantasmatiquement le traducteur et son auteur. Il est aisé d'imaginer, en effet, que lorsqu'un traducteur voue la majeure partie de son travail au même auteur, la frontière psychologique qui sépare habituellement le traducteur de l'écrivain tend à s'estomper. Ce type d'entreprise, qui ne se conçoit, à l'origine, qu'animée par un sentiment de reconnaissance des affinités entre l'autre et soi, renforce encore, à mesure qu'elle prend forme, l'identification fantasmatique. On ne peut qu'être troublé par ce constat : Pavans n'a pas traduit un premier récit de James, qui en aurait entraîné un autre, puis un autre encore jusqu'à ce qu'une somme se constitue. D'emblée, il s'est posé comme celui qui voulait tout traduire d'un ensemble de textes - devenir, à partir des années quatre-vingt, la voix française, reconnaissable d'un récit à l'autre, de Henry James.

Nous disposons de deux documents de nature différente pour comprendre l'esprit dans lequel le projet s'est formé. La « Présentation » ou préface de Pavans au premier tome des Nouvelles complètes (1864-1975)17 et son article de 1993 relatif à l'ordre des mots de la phrase jamesienne ${ }^{18}$.

15. Ces informations m'ont été fournies par Jean Pavans lui-même dans la correspondance et les conversations que nous avons échangées ces dernières années.

16. Rares sont, de nos jours, les exemples comparables. Georges-Arthur Goldschmidt s'est, en dehors de ses propres écrits, attaché à la traduction des livres de Peter Handke ; Claire Cayron, quant à elle, ne se définissait pas « comme traductrice [...], ni même comme traductrice du portugais, mais comme la traductrice de Miguel Torga » (« Itinéraire d'une traduction », Actes des premières assises de la traduction littéraire (Arles, 1984), Arles, Actes Sud/Atlas, 1985, p. 88). Jean-Michel Déprats, avec ses traductions de William Shakespeare, est un troisième exemple.

17. Publiées aux éditions de la Différence, en 1990.

18. « Rythme, reliefs et niveaux des phrases initiales de trois fictions de Henry James », Palimpsestes, $\mathrm{n}^{\circ} 7$, « L'ordre des mots », dir. Paul Bensimon, 1993, p. 85-91. 
$\mathrm{La}$ « Présentation », d'une vingtaine de grandes pages, s'ouvre sur la mise en exergue d'une citation de T.S. Eliot, compatriote de James à plus d'un titre puisque, Américain comme lui, Eliot avait choisi de s'établir en Angleterre et de devenir citoyen britannique. Cette citation est extraite d'un essai sur Henry James publié par Eliot en 1924 : « Une chose est certaine : les livres de Henry James forment un tout. Il est nécessaire de les lire tous, car il faut en saisir, si l'on doit en saisir quelque chose, à la fois l'unité et la progression. Le développement graduel et la fondamentale identité d'esprit sont également importants, et leur leçon est une » (p. 7).

Selon Walter Benjamin, une œuvre, tout en ne « désirant » pas nécessairement sa traduction, porte en elle son mode de traduction spécifique qu'il appartient au traducteur de mettre au jour. Jean Pavans retrouve ici les conséquences directes de cette approche de la tâche du traducteur. C'est parce qu'ils « font œuvre » que les livres de Henry James appellent non seulement le projet d'une "intégrale », mais aussi celui d'une unité de ton dans la traduction. Dès l'exergue, donc, Pavans inscrit son entreprise dans une saisie globale de l'œuvre jamesienne. Par ce geste, il rompt avec l'histoire des traductions qui ont précédé la sienne, et innove. Les traductions antérieures, en effet, ont en commun leur morcellement et, dans les rares cas où une unité s'est, tant bien que mal, dessinée, elle a été le fruit d'une politique éditoriale thématique : on a ainsi regroupé en volume(s) plusieurs nouvelles où l'on estimait forte la présence de l'élément fantastique, ou bien qui mettaient en scène des destins féminins ${ }^{19}$. Aucun de ces regroupements, cependant, n'est apte à rendre compte du double mouvement d'unité et de progression qui s'opère dans l'écriture. D'où l'importance qu'un traducteur s'attache à la totalité d'un corpus.

Quelques paragraphes plus loin, Jean Pavans expose et justifie son entreprise de façon encore plus explicite :

Si l'idée d'une « intégrale » de Henry James s'impose depuis longtemps, elle n'a jamais été vraiment accomplie, et, à cet égard, il faut reconnaître que la situation, à ce jour, pour les traductions françaises n'est guère plus confuse ou dispersée que la situation pour les originaux dans les pays anglo-saxons, Angleterre ou États-Unis. Il est vrai que James avait quelque peu compliqué le problème en faisant lui-même un choix pour établir une édition " complète » et révisée, où il ne retint que la moitié de ses nouvelles, pas tous ses romans, et qui parut à New York en vingt-quatre volumes, de 1907 à 1917, selon une organisation plus thématique que chronologique - scènes de la vie internationale, scènes de la vie anglaise, scènes de la vie artistique ou littéraire, histoires surnaturelles, etc. -, car l'auteur avait alors en tête Balzac et le modèle vénéré de La Comédie humaine, avec la superstition d'aboutir au même nombre de volumes que celui d'une édition qu'il possédait, nombre qui toutefois se trouva dépassé. En outre, il avait tout au long de sa carrière fait paraître de nombreux recueils de certaines de ses nouvelles, avec là également des révisions.

Or, dans les années soixante, un demi-siècle, donc, après la mort de James, Leon Edel (si les textes originaux sont une bible pour les jamesiens, les travaux de Leon Edel, en particulier son admirable biographie qui est peut-être le plus beau monument critique élevé à un écrivain, sont leur catéchisme) - Leon Edel publia en douze volumes une intégrale des nouvelles, dans leur ordre chronologique, de

19. Ainsi de Histoires de fantômes (Sir Edmund Orme, Le Coin plaisant), traduit par Louise Servicen aux éditions Aubier-Flammarion en 1970 ; ou de Les Deux Visages (L'Auteur de Beltraffio, L'Humiliation des Northmore, Le Château de Fordham, Une tournée de visites), traduit par Diane de Margerie et François-Xavier Jaujard aux Lettres Nouvelles, en 1977. 
laquelle de nombreux traducteurs et éditeurs français ont depuis extrait des recueils plus ou moins homogènes. C'est cette publication, hélas ! aujourd'hui épuisée et qui ne se trouve qu'en bibliothèque, que nous suivons aussi fidèlement que le permet l'exercice périlleux de la traduction.

Bon nombre d'informations essentielles nous renseignent ici sur le projet du traducteur. Il $\mathrm{y}$ a, tout d'abord, le rappel de la confusion qui règne dans l'édition française des nouvelles - une confusion à l'image de l'édition américaine quelque peu compliquée par les sélections spécifiques que James a lui-même opérées et, d'une manière générale, le puissant contrôle qu'il a toujours entendu exercer sur le destin de son œuvre ${ }^{20}$. La première tâche du traducteur, dans ces conditions, est de surmonter le verrouillage opéré par l'auteur, et quel moyen plus judicieux de le faire que de s'en remettre à un choix tiers, et qui plus est, autorisé ? D'où la référence au grand spécialiste de James, Leon Edel - dans une phrase de plus de quatre-vingts mots, truffée d'incises et de retours sur elle-même, qui n'est pas sans rappeler le style jamesien lui-même !

La suite de la préface est consacrée à une présentation chronologique des vingt-sept nouvelles que comporte le volume - textes écrits entre 1864 et 1875, dont bon nombre sont ici traduits pour la première fois. Pavans en présente la teneur : il résume la matrice thématique (quand elle est résumable, ce qui est encore majoritairement le cas, à ce stade de la carrière de James) et propose certaines interprétations, dans lesquelles s'entendent des échos de l'abondante critique jamesienne mêlés d' " anticipations » personnelles, toujours avancées avec prudence. Ainsi conclut-il d'un récit de 1865, L'Histoire d'une année (The Story of the Year) :

Remarquons en passant, quoique ce soit beaucoup anticiper, que James a presque constamment considéré les rapports amoureux comme vampiriques [...]. Souvent, dans les fictions de James, si c'est l'homme qui cède, il meurt tout simplement; si c'est la femme, elle se vide de sa substance, à une époque, peutêtre, qui se prêtait à ce point de vue. On peut toujours se dire que James ne faisait ainsi que se convaincre de la sécurité du célibat, et même du danger mortel d'une liaison. En quelque sorte, toute son œuvre, sinon la vie, lui donne raison. Notons aussi [...] qu'il concevait les liaisons essentiellement en terme de mariage - vais-je, ne vais-je pas l'épouser ? ou bien : quelle est la fêlure propre à tout mariage ? Tout tourne autour de cela, surtout si les personnages impliqués sont américains - écho sans doute du puritanisme. Parfois, enfin, l'agneau du sacrifice sur l' « autel de l'amour » n'est aucun des deux conjoints ; c'est alors un enfant, et sans aller jusqu'aux exemples particulièrement cruels et émouvants de L'Élève (1891) et de L'Auteur de Beltraffio (1884), nous trouverons ici le frôlement de ce thème « infanticide » dans Mon ami Bingham et dans Un problème (p. 12).

Ces observations présentées comme incidentes (« en passant») sont exemplaires du type de regard critique que porte Jean Pavans sur l'œuvre qu'il traduit. Sans cesse, chez lui, l'intelligence des fictions oscille entre l'éclairage biographique - ici la prédilection de James pour le célibat et la confirmation de certaines intuitions par leur extension à l'ensemble de l'œuvre. Le choix

20. Je me permets de renvoyer à mon article « Henry James dans l'atelier », dans L'Atelier du roman, $\mathrm{n}^{\circ} 5$, 1995, $^{\prime}$ p. 161-167. On y verra, en particulier, que le don de l'œuvre, chez James, n'est jamais entièrement consenti et s'accompagne toujours d'une forme quelconque de rétention. Le « donner/reprendre » s'étendait d'ailleurs jusqu'aux traductions elles-mêmes, comme en témoigne la correspondance avec Auguste Monod, le premier des traducteurs français de l'écrivain. 
de l'éclairage biographique, de la part d'un traducteur qui n'ignore pas l'existence d'autres postures critiques (celles, notamment, qui s'adressent directement à l'écriture), constitue un indice supplémentaire de l'étroitesse des liens que Pavans a noués, par-delà l'œuvre, avec la personnalité même - on serait presque tenté de dire, la personne - de son auteur.

Si la « Présentation » de Pavans comporte ici et là des considérations relatives au type de lecture qui a orienté le travail, elle s'abstient de tout commentaire quant à la traduction ellemême, hormis la remarque que l'exercice est périlleux (il n'est pas dit, toutefois, qu'il soit plus périlleux que n'importe quel autre exercice de traduction). C'est en fait dans l'article qu'il consacre, en 1993, au problème de l'ordre des mots que Pavans précise la façon dont il conçoit et mène son travail : « [...] ce qui est frappant dans le style si particulier de Henry James est que la phrase achoppe rarement aux mots, que les mots semblent toujours pris dans le mouvement de la phrase, de sorte que le traducteur, toutes autres difficultés mises à part, et elles sont célèbres et réelles, trouve toute naturelle la transposition, et obtient aisément, par la simple fidélité, des phrases qui paraissent obéir aux idiosyncrasies de la langue française. » $\mathrm{Ce}$ mouvement si particulier de la phrase jamesienne, Pavans l'illustre en analysant l'incipit du roman de 1902, The Wings of the Dove, que je rappelle ici pour mémoire :

She waited, Kate Croy, for her father to come in, but he kept her unconscionably and there were moments at which she showed herself, in the glass over the mantel, a face positively pale with the irritation that had brought her to the point of going away without sight of him.

Suit un commentaire sur l'attaque de cette phrase inaugurale et l'effet de dédoublement qu'elle produit :

Ce « she » et ce « Kate Croy » nous donnent dans la phrase une double figure d'une héröne qui ellemême regarde son double dans un miroir et qui, en étant peut-être ainsi rappelée à la conscience de sa duplicité, nous la fait vaguement soupçonner, à nous lecteurs. Aussi est-il primordial de respecter ce cri extérieur ou ce reflet, « Kate Croy », et se dérober à la phrase telle qu'elle se présente, traduire par « Kate Croy attendait son père », est une trahison non seulement parce que c'est une platitude, mais aussi parce que c'est refuser de suivre un grand écrivain dans ses audaces les plus significatives, et se ranger du côté d'hostilités, d'incompréhensions, dont lui-même eut beaucoup à souffrir (p. 88-89).

Pavans énonce ici son principe de traduction : suivre la phrase dans son agencement propre. Dans l'exemple choisi, cela implique de reproduire l'effet d'étrangeté (au sens propre : l'effet de langue étrangère, de présence en filigrane de la langue française à l'intérieur de l'anglais) introduit par le redoublement du sujet. Mais de ce principe découlent un certain nombre de conséquences dont la somme constitue ce qu'Antoine Berman a appelé le " projet de traduction ${ }^{21} »$. Ici, par exemple, la discrète réfutation - elle n'est pas explicitement nommée

21. A. Berman, Pour une critique des traductions, op. cit. : « Toute traduction conséquente est portée par un projet, ou [une] visée articulée. Le projet ou [la] visée sont déterminés à la fois par la position traductive et par les exigences à chaque fois spécifiques posées par l'œuvre à traduire. Le projet définit la manière dont, d'une part, le traducteur va accomplir la translation littéraire, d'autre part, assumer la traduction même, choisir un "mode" de traduction, une "manière de traduire" [...]. Tout ce qu'un traducteur peut dire et écrire à propos de son projet n'a réalité que dans la traduction. Et cependant, la traduction n'est jamais que la réalisation du projet : elle va où la mène le projet, et jusqu'où la mène le projet. Elle ne nous dit la vérité du projet qu'en nous révélant comment il a été réalisé (et non, finalement, s'il a été réalisé) et quelles ont été les conséquences du projet par rapport à l'original » (p. 76-77). 
- de la seule traduction en langue française publiée à ce jour : celle, parue en 1947, de Marie Tadié, auteur de la « platitude » « Kate Croy attendait son père22 ». Par-delà la réfutation, c'est de nouveau son lien étroit à Henry James qu'affirme Pavans : lui ne le trahira pas, si par trahison l'on entend la banalisation, au profit du « bon français » qu'on nous enseigne à l'école, d'une écriture qui rompt avec l'usage grammatical de l'anglais. S'il faut choisir un camp («se ranger »), que ce ne soit pas celui des « hostilités » et « incompréhensions » qui blessaient l'écrivain - critiques perpétuelles de son frère William ${ }^{23}$, accusations de " maniérisme », voire de préciosité qui établirent Henry James dans une réputation d'écrivain difficile à lire, donc à traduire. Au reste, comme pour rappeler que le projet de traduction ne voit sa validité confirmée que dans le texte traduit lui-même, Pavans propose sa propre traduction de l'incipit :

Elle attendait, Kate Croy, l'entrée de son père, mais il la retenait déraisonnablement, et il y avait des moments où elle montrait au miroir de la cheminée un visage absolument livide de l'irritation qui l'avait conduite au point de s'en aller sans l'avoir vu (p. 90).

Non seulement cette traduction illustre parfaitement le principe de respect de l'agencement de la phrase tel que Pavans vient de l'exposer, mais elle retrouve l'effet dilatoire produit par la phrase anglaise, notamment grâce à l'abondance des virgules et la présence de segments tels que le peu banal «there were moments at which» ou «that had brought her to the point of »). D'une phrase anglaise dans laquelle l'écriture est manifestement accordée à la situation décrite - une attente qui se prolonge jusqu'à devenir irritante - le traducteur a fabriqué une phrase française dont le mot à mot produit le même effet de ralentissement et d'irritation. La cohérence est donc vérifiée, tant au niveau du principe de traduction que du projet global de traduction et de la fidélité à l'entreprise esthétique de l'écrivain.

On voit donc quelle mine de renseignements recèlent, pour l'étude du processus traductif, ces deux entours du texte traduit que sont la «Présentation » de Jean Pavans et son article de 1993 - que l'on pourrait utilement compléter de deux autres « paratextes »: Le Sceau brisé, fiction dans laquelle Pavans pastiche le style jamesien, et son adaptation pour l'opéra de La Bête dans la jungle. Ce sont là des apports au dossier génétique d'autant plus précieux que manquent, en l'espèce, d'autres éléments importants tels que les divers états qui ont permis d'aboutir à la traduction publiée.

L'absence assez courante, comme nous l'avons vu, de «brouillons » de traduction incite le généticien à rechercher les traces du processus ailleurs, dans d'autres lieux. La lecture que fait le traducteur du texte à traduire, dont les traces peuvent subsister sous forme d'annotations dans les marges de l'original, par exemple, ou de soulignements, est à cet égard un lieu particulièrement foisonnant.

22. Les Ailes de la colombe, trad. Marie Tadié, Paris, Laffont, 1947. Voici la phrase dans son entier : « Kate Croy attendait son père. Elle l'avait même tant attendu que la glace de la cheminée lui renvoyait par instants l'image d'un visage tout pâle de l'irritation qui la poussait presque à partir sans l'avoir vu. »

23. La correspondance échangée entre les deux frères au fil des années montre que William a maintes fois usé de ses prérogatives de frère aîné et de philosophe pour inciter Henry à une écriture plus « simple », s'étonnant même, ici et là, que Henry puisse trouver des lecteurs enthousiastes. 


\section{Lire pour traduire}

Dans « Écrire la lecture », l'un des textes brefs qui composent Le Bruissement de la langue, Roland Barthes interpelle le lecteur en ces termes :

Ne vous est-il jamais arrivé, lisant un livre, de vous arrêter sans cesse dans votre lecture, non par désintérêt, mais au contraire par afflux d'idées, d'excitations, d'associations ? En un mot, ne vous est-il pas arrivé de lire en levant la tête ?

Voici qu'aux formes de lecture classiquement répertoriées - silencieuse (avec les yeux), à voix haute (accompagnant le repas des moines), murmurée (avec ce mouvement caractéristique des lèvres qui instituera la rumination comme l'une des grandes métaphores de l'activité intellectuelle) - s'ajoute une quatrième forme, plus facétieuse 24 . À bien y songer, en effet, cette lecture s'oppose corporellement à la lecture le nez plongé ou, mieux encore, « fourré » dans les livres qui, selon l'imagerie populaire, signale l'érudit. Distraite en apparence, cette lecture la tête levée n'en est pas moins pleinement productive : non seulement d'associations d'idées ou de mots, de réminiscences fugaces et d'échos lointains, mais aussi, dans l'expérience qu'en a Barthes, de l'écriture même : «Qu'est-ce donc que S/Z ? Simplement un texte, ce texte que nous écrivons dans notre tête quand nous la levons. »

Barthes décrit ici le type même de lecture qui est aussi celle du traducteur, et dont le texte traduit fixe provisoirement - aucune traduction ne peut jamais être dite « définitive »la nature. La traduction apparaît donc comme une forme particulière, modifiée de la lecture, à telle enseigne que l'examen minutieux d'une traduction nous renseigne précisément sur la lecture que le traducteur a faite de l'ouvre.

Le traducteur lève la tête à tout moment : dès sa toute première lecture de l'original, qui est déjà faite en vue de la traduction. On pourrait sans doute rapprocher ce mode de lecture de celui du critique pour lequel même la lecture primitive d'un texte est rarement « innocente ». Mais l'analogie la plus pertinente me semble être avec l'interprète musical. L'instrumentiste est devant sa partition comme le traducteur devant son original : il cherche à la comprendre en vue de la musique. On dit qu'il la déchiffre (de fait, elle est mathématiquement codée), ce qui suppose qu'il en repère les divers traits stylistiques, les identifie comme propres à cette écriture-là, cette langue du musicien qu'il devra comprendre s'il veut l'interpréter. Le traducteur qui découvre l'original bute sur un mot, un segment de phrase, éprouve confusément un rythme, une unité de base (la phrase, chez certains écrivains, le paragraphe chez d'autres), l'enchaînement de sonorités dans une séquence, la récurrence de certaines images, la prédilection pour telle ou telle syntaxe, etc. Quel que soit ce qui lui fait lever la tête, interrompre le cours de sa lecture - et qu'il en marque ou non précisément la trace - le traducteur lit en prétraduisant.

Antoine Berman avait tôt pressenti le caractère spécifique de la lecture du traducteur. Il y consacre une note dans son ouvrage de 1984, L'Épreuve de l'étranger :

On pourrait dire que l'analyse textuelle à laquelle un traducteur doit se livrer - comme repérer dans un roman le réseau des termes et des associations fondamentaux, le « système » de son écriture, etc. - est a priori déterminée par le fait qu'il va traduire : lire pour traduire, c'est illuminer le texte

24. Barthes la dit «à la fois irrespectueuse, puisqu'elle coupe le texte, et éprise, puisqu'elle y revient et s'en nourrit ». 
lolloped its way back into the water in the last hour or two.

Petroc was not a chatterbox like Hedley or Morwenna or strong and silent (for which read sulky) like Garfield. He talked if he felt like it but more often he was too selfcontained to bother. In this he strongly resembled Antony, so that loving him was tied up in loving his father. Secretly he reminded her of the best kind of dog; amusing himself while always keeping half an eye on his owner. While he raced about at the water's edge she kicked off her espadrilles, which had never been the same since she mistakenly stood in a puddle at the fishmonger's in them. She crossed the beach to the first cave, where she changed swiftly into her bathing costume, and left the picnic in the shade to keep it cool. There was a stream which splashed down from the valley above even at this dry time of year. She slipped their bottle of apple juice into one of the pools it made, hoping to chill it. Batance

One of the reasons this beach was special was the $\delta$ violent changes wrought on it from/day to/day and/season to season. Sometimes the/sand/was heaped to one side, sometimes to the other. Sometimes the stream created a deep, winding gorge through the sand/down which/dogs and children delighted in slithering. Sometimes it carved a stealthy path feet beneath the beach surface and was undetectable until the point where it spilled out into the surf. Sometimes she/would/find the/sand clean/and pure, as she/had today. At other times it would be laden with fascinating junk washed from passing ships - rubber shoe soles, plastic bottles, wrecked packing cases and once, to Garfield's and Hedley's delight, some yachtsman's ingen-

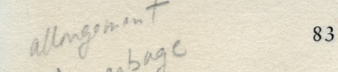

83

Fig. 2

d'une lumière qui n'est pas de l'ordre de l'herméneutique seulement, c'est opérer une lecturetraduction - une prétraduction. Cette prétraduction peut apparaître si l'on regarde les mots, les phrases ou les segments de phrases qu'un traducteur a soulignés dans l'ouvrage à traduire avant de commencer la traduction proprement dite : non seulement les passages qu'il ne « comprend » pas (que l'on supposera peu nombreux), mais ceux qui, à la première lecture, posent un problème de traduction à cause de leur grande distance par rapport à la « langue d'arrivée ». On a là les lignes de crête de l'étrangeté de l'œuvre, ou sa ligne de résistance à la traduction. Et cette ligne coïncide en grande partie avec le système original de l'œuvre dans sa langue. De là, est possible une certaine lecture de l'œuvre, qui peut se transformer en lecture « critique ». Traduire est en ce sens une connaissance de l'œuvre25.

Cette lecture ne laisse souvent pas d'autre trace que la traduction même à laquelle elle aboutira, mais il existe parfois des documents qui en portent le témoignage. Mon exemplaire du roman de Patrick Gale, Notes from an Exhibition (2007), que j'ai traduit en 200926, a conservé l'empreinte de ce qui m'avait immédiatement frappée à la lecture : un balancement de la phrase, au chapitre IV, qui m'évoquait spontanément le flux et reflux des vagues dans le «poème dramatique » de Virginia Woolf, The Waves (1931). Le paragraphe annoté (fig. 2) décrit la perception qu'a le personnage central du roman, Rachel Kelly, de la plage de Cornouailles où elle est venue passer une journée avec son jeune fils Petroc. Rachel Kelly étant peintre, c'est un regard particulièrement attentif au détail qu'elle porte sur le paysage un paysage que le lecteur découvre à mesure qu'elle-même le fixe par le dessin.

25. Antoine Berman, L'Épreuve de l'étranger. Culture et traduction dans l' Allemagne romantique, Paris, Gallimard, 1984, p. 248-249.

26. Patrick Gale, Tableaux d'une exposition, trad. Fabienne Durand-Bogaert, Paris, Belfond, 2009. 
Quatre types d'annotations, portées au crayon à papier, figurent sur cet extrait : des traits obliques, insérés à plusieurs endroits comme autant de séparations entre les mots du texte ; des soulignements affectant certains termes ; des groupes de lettres entourés ; enfin, quelques annotations en marge du texte ou en bas de page. L'ensemble de ces annotations n'a pas été porté dès la première lecture - celle que je fais, à chaque fois qu'un éditeur me propose une traduction, de l'ensemble du texte pour savoir s'il m'intéresse ou non de le traduire. De cette lecture primitive, silencieuse, je ne souhaite en effet conserver que des traces immatérielles, une impression ou une sensation générale, même si je ne cesse d'anticiper mentalement les difficultés auxquelles je serai exposée au cours de la traduction proprement dite. La deuxième lecture est celle que j'effectue de chaque chapitre préalablement à sa traduction, et c'est en général pendant cette phase-là que j'annote. Mais dans l'exemple ci-dessus, les annotations furent portées à la troisième lecture : au cours de la deuxième, en effet, j'avais été sensible à une qualité poétique particulière de l'écriture (qui ne caractérise pas l'ensemble du roman), dans laquelle le rythme des phrases et les sonorités me paraissaient accordés, de façon woolfienne, à la perception du paysage. D'où une troisième lecture, à voix haute cette fois, au cours de laquelle les annotations portées le furent en vue de confirmer cette intuition.

La première phrase - "One of the reasons this beach was special was the violent changes wrought on it from day to day and season to season »-, lue à voix haute, fait entendre, du fait de l'accentuation propre à la langue anglaise, une sorte de rumeur qui enfle et culmine avec le segment sonore violent changes wrought on it avant de retomber selon un rythme dans lequel alternent brèves et longues (from day to day and season to season), le dernier segment (season to season) imprimant à la phrase un effet d'étirement. Le ton est donné, qui a guidé mes annotations. Les traits obliques isolent les monosyllabes (comme dans la phrase : "Sometimes she/would/find/the/sand/clean/and/pure/as/she/had/ today ») - monosyllabes dont la fréquence diminue nettement dès lors que le texte décrit le rebut (junk ou garbage) dont se sont délestés les bateaux de passage : à l'énumération de ce qui est en trop correspond un allongement des mots produisant une rupture de rythme (d'où mon annotation « allongement $\Rightarrow$ garbage). Les groupes de lettres entourés signalent la récurrence de certains sons ( $i$ long : beach, season, stream, deep, beneath, clean) ou d'une même graphie (le ea diphtongué de created ou prononcé [é] de stealthy). Enfin, le flux et le reflux sont scandés par le mot sometimes, souligné quatre fois, et dont la disparition, au profit de la locution At other times, à la dixième ligne du paragraphe, constitue un autre indice du changement de rythme et de registre qui s'opère à cet endroit du texte.

Par le geste d'annoter ce paragraphe, que je plaçai sous le signe du «balancement » (sans doute le premier terme qui me vint à l'esprit, même si je le trouve aujourd'hui imprécis), je m'assurai de la validité d'une partie de mon hypothèse : j'étais bien en présence, en cet endroit du texte, d'une prose poétique, d'une de ces « zones signifiantes où une œuvre atteint sa propre visée (pas forcément celle de l'auteur) et son propre centre de gravité27 ». Mais l'autre partie, la réminiscence woolfienne, me restait à confirmer. Je fis donc retour à mon exemplaire de The Waves (fig. 3), que j'avais annoté, ou plutôt surligné, en une autre occasion pour les besoins de mon enseignement à l'École des hautes études.

27. Antoine Berman, Pour une critique des traductions, op. cit., p. 70. 
The sun* had not yet risen. The sea was indistinguishable from the sky, except that the sea was slightly creased as if a cloth had wrinkles in it. Gradually as the sky whitened a dark line lay on the horizon dividing the sea from the sky and the grey cloth became barred with thick strokes moving, one after another, beneath the surface, following each other, pursuing each other, perpetually.

As they neared the shore each bar rose, heaped itself, broke and swept a thin veil of white water across the sand. The wave paused, and then drew out again, sighing like a sleeper whose breath comes and goes unconsciously. Gradually the dark bar on the horizon became clear as if the sediment in an old wine-bottle had sunk and left the glass green. Bebind it, too, the sky cleared as if the white sediment there had sunk, or as if the arm of a woman couched beneath the borizon had raised a lamp and flat bars of white, green and yellow spread across the sky like the blades of a fan. Then she raised her lamp higher and the air seemed to become fibrous and to tear away from the green surface flickering and flaming in red and yellow fibres like the smoky fire that roars from a bonfire. Gradually the fibres of the burning bonfire were fused into one baze, one incandescence which lifted the weight of the woollen grey sky on top of it and turned it to a million atoms of soft blue. The surface of the sea slowly became transparent and lay rippling and sparkling until the dark stripes were almost rubbed out. Slowly the arm that beld the lamp raised it

Fig. 3

Une simple saisie visuelle de cette page d'ouverture de The Waves suffit à faire apparaître les correspondances avec l'extrait du roman de Patrick Gale : même type de balancement dû à l'alternance des monosyllabes sky/sea/sun (ce dernier faisant lien entre les deux autres) ; même type de scansion, à cette différence près que l'adverbe n'est pas sometimes, mais gradually - répété trois fois, en ouverture d'une phrase décrivant les variations de la lumière (là où Gale décrit le mouvement du sable); même ondulation de la phrase - amplification, crête, retombée - dans la description des stries qui barrent la surface de l'eau chez Woolf : "As they neared the shore each bar rose, heaped itself, broke and swept a thin veil of white water across the sand. » Et de même que at other times se substitue, chez Gale, à sometimes, slowly vient, chez Woolf, compléter gradually et affiner la temporalité.

Une analyse plus approfondie ferait certainement apparaître bien d'autres correspondances, mais ce qui importe ici n'est pas d'en fournir une liste exhaustive : elles valent essentiellement comme traces de la sédimentation qui s'était effectuée en moi et dont je n'ai pris conscience que dans cette phase du prétraduire (et grâce à elle). Les écrivains écrivent avec d'autres écrivains, Gale avec Woolf, Woolf avec Joyce28, Joyce avec (au

28. Comme l'a montré Daniel Ferrer dans « Les bibliothèques virtuelles de James Joyce et de Virginia Woolf », dans Bibliothèques d'écrivains, Paris, Éditions du CNRS, 2001, p. 171-193. 
moins) Homère, etc. De même pour les traducteurs : leur pratique fait revenir des souvenirs de lecture, réactive la mémoire de descriptions ou de scènes romanesques oubliées, réveille des zones de langage endormies. Ils ne sont au fond pas si solitaires qu'on le dit, car leurs livres (de départ, d'arrivée) sont bien peuplés. Il ne m'appartient pas de dire si ma traduction du passage de Notes from an Exhibition 29 conserve quelque chose de la discrète présence de Woolf à mes côtés, mais nul doute que mon travail fut soutenu par le sentiment d'une affinité entre elle et Patrick Gale.

Revenons un instant à Barthes, qui semble regretter, dans le texte auquel je fais référence plus haut, l'importance « exorbitante » qu'on accorde à l'origine de l'œuvre, au détriment de sa destination. Personne, déclare-t-il, ne «s'intéresse au lieu où l'œuvre va et se disperse $30 »-$ par quoi il faut entendre, se fragmente en autant de microlectures individuelles qu'il y a de lecteurs. L'une des destinations de l'œuvre est assurément la traduction qui, comme l'a suggéré Walter Benjamin, est une forme de vie de l'œuvre, l'une des phases de son processus vital ${ }^{31}$. Si elle s'y disperse, c'est au sens d'une dissémination fructueuse, car ce qu'a semé l'œuvre se récolte, et donc se rassemble en une autre langue, un autre texte. Le généticien, grand rassembleur des traces du processus vital, constituera-til son dossier de la même façon selon qu'il s'applique au travail du traducteur ou à celui de l'écrivain ? À cette question, la réponse ne peut être que prudente et nuancée. Étapes et procédures
génétiques
Si la phase "prétraductive » au cours de laquelle le traducteur réunit de la documentation, annote son original ou procède à l'examen de ce que sa lecture a déposé en lui peut se comparer à la phase prérédactionnelle repérable chez l'écrivain, la suite du processus tend à se singulariser. On ne trouvera guère, chez les traducteurs, de "plans développés » ou d'« esquisses générales ». Lorsqu'il en subsiste des traces matérielles, la phase « rédactionnelle » de la traduction, pour l'appeler ainsi, prend d'emblée la forme d'un brouillon, dont la physionomie diffère selon la façon particulière dont le traducteur travaille. Certains traducteurs ont besoin de faire un premier « jet» de l'ensemble du texte à traduire, avant d'y revenir, aussi souvent que nécessaire, jusqu'à ce qu'ils estiment leur traduction aboutie, c'est-à-dire apte à quitter leur atelier pour être remise à l'éditeur. D'autres, en revanche, ne peuvent progresser dans leur travail en laissant derrière eux une phrase, une tournure, voire un simple mot perçus comme approximatifs : ceux-là reviennent sur leur traduction à chaque coin de phrase, la modifiant à mesure et parfois de façon

29. «Cette plage avait ceci d'exceptionnel qu'elle était, au fil des jours et des saisons, soumise à des changements violents. Le sable s'accumulait tantôt d'un côté, tantôt de l'autre. Tantôt le courant sculptait dans le sable une gorge profonde et sinueuse où les enfants et les chiens aimaient batifoler. Tantôt il ravinait sournoisement la surface de la plage, y creusant un sillon dont l'existence ne se révélait qu'à la jonction avec l'écume. Tantôt le sable était propre et vierge, comme aujourd'hui. Tantôt il était jonché de toutes les saletés fascinantes dont les bateaux de passage se délestaient : semelles en caoutchouc, bouteilles en plastique, caisses d'emballage, voire, à l'immense joie de Garfield et de Hedley, la poulaine qu'un marin astucieux avait lui-même bricolée en combinant un couvercle de WC en bois d'acajou avec une vieille chaise de salle à manger » (Tableaux d' une exposition, op. cit., p. 88). 30. Je souligne.

31. «La traduction est plus tardive que l'original, et pour les œuvres importantes, qui ne trouvent jamais le traducteur élu à l'époque de leur surgissement, elle marque le stade de leur survivance », dans Walter Benjamin, «La tâche du traducteur », trad. Martine Broda, Po\&sie, n 55, 1991, p. 151. 
significative, et ne la retouchant que très peu lors de la relecture finale. D'autres encore mixent les deux manières : ils font un premier « jet » d'une partie du texte à traduire, un chapitre par exemple, y reviennent et le modifient avant de poursuivre. Les « maniaques du polissage », pour reprendre la formule de Jean-Louis Lebrave ${ }^{32}$, comme les adeptes du «flux » se trouvent donc aussi chez les traducteurs.

Un brouillon de traduction, pris qu'il est entre le texte « original » et la version imprimée de la traduction, exige du généticien un effort particulier pour suspendre ses conclusions. Il est tentant, en effet, de se référer constamment aux deux bouts de la chaîne, et ainsi de faire du brouillon une lecture déterminée par les points de départ et/ou d'arrivée. Dans l'idéal, le généticien devrait s'efforcer, pour un temps, d'oublier ces deux termes pour se concentrer exclusivement sur ce qui se passe dans l'espace du brouillon lui-même, autrement dit les données identifiables comme traces de l'activité de traduction. Elles ne sont pas différentes de celles auxquelles nous a habitués la génétique textuelle : ajouts, suppressions, biffures, becquets d'insertion, flèches signalant le déplacement d'un mot ou d'un groupe de mots, etc. Mon intuition - mais elle devra être mise à l'épreuve des brouillons eux-mêmes - est que des quatre grandes opérations que sont l'ajout, la suppression, le déplacement et le remplacement, celle à laquelle le traducteur s'adonne le moins volontiers est la suppression. En effet, plus le traducteur a intériorisé l'idée qu'il « sert » le texte, moins il s'autorise à en retrancher certains éléments : la hantise de la déperdition non seulement de sens, mais tout simplement de mots guette toujours. Quel traducteur réussit à se départir tout à fait du vieux rêve de faire coïncider chaque mot ou expression du texte de départ avec un mot ou une expression dans le texte d'arrivée ? Jean-René Ladmiral a théorisé ce qu'il décrit comme le "mécanisme de feed-back herméneutique ", autrement dit la phase où le traducteur revient au texte source (ce sont ses termes) pour vérifier que le texte cible lui « correspond » bien. Or c'est bien lors de cette étape que le danger de réduire l'acte de traduire à une simple opération sur la langue est le plus grand, le réflexe du traducteur, souvent complété de celui du réviseur, étant de vérifier que les mots du texte source ont bien trouvé leur cible dans la traduction (au risque d'oublier que ces mots doivent faire texte). Ils la trouvent, dans les traductions méticuleuses, et même souvent en excès, si l'on en juge par la remarque de Berman selon laquelle « toute traduction est tendanciellement plus longue que l'original » :

Mais cet allongement, du point de vue du texte, peut bien être qualifié de «vide » et coexister avec diverses formes quantitatives d'appauvrissement. Je veux dire par là que l'ajout n'ajoute rien, qu'il ne fait qu'accroître la masse brute du texte, sans du tout augmenter sa parlance ou sa signifiance. Les explicitations rendent peut-être l'œuvre plus « claire », mais obscurcissent en fait son propre mode de clarté. L'allongement, en outre, est un relâchement portant atteinte à la rythmique de l'œuvre. C'est ce qu'on appelle souvent la « surtraduction », dont un cas typique est le Moby Dick d'Armel Guerne. Moby Dick « allongé », d'océanique, devient boursouflé et inutilement titanesque 33 .

32. "Lire et écrire au XXI ${ }^{\mathrm{e}}$ siècle », dans J. Anis et N. Marty, Lecture-écriture et nouvelles technologies [Journée académique du 10 mai 2000 à l' initiative de l'académie de Paris et de l'université de Paris-X Nanterre], Paris, CNDP, 2000, p. 13-22.

33. Antoine Berman, «La traduction et la lettre ou l'Auberge du lointain », dans Les Tours de Babel, Mauvezin, T.E.R, 1985, p. 71 (rééd. Paris, Éditions du Seuil, 1999). Notons que Berman classe l'allongement parmi les tendances « déformantes » de la traduction. 
Devant un brouillon de traduction, trois options semblent possibles : soit l'état considéré apparaît comme un texte renié (cas sans doute le plus fréquent, mais la question se posera alors de savoir à qui imputer cette mise au rebut); soit le chercheur le traite comme un texte virtuel, soit encore les deux ensemble (un texte virtuel mis au rebut). Dans "Lire et écrire au Xxi ${ }^{\text {e }}$ siècle », Jean-Louis Lebrave évoque tout ce que " le texte définitif linéaire enlève de possibles au texte en gestation dont il procède ». À l'inverse, l'acte de traduction, qui opère lui aussi de façon linéaire - seul moyen de préserver le tissu du texte - rajoute, pendant un temps au moins, des possibles au texte dont il procède. Pour le dire autrement, il est une phase dans laquelle le texte source, bien que « définitif » (stabilisé), reste un hypertexte pour le traducteur : il est susceptible de multiples significations, qui sont autant d'embranchements possibles entre lesquels le traducteur devra opérer des choix. Sans prendre l'exemple extrême de la traduction d'un écrivain tel que Joyce, où chaque bifurcation dans laquelle le traducteur s'engage n'est qu'un renoncement à d'autres voies également possibles, c'est là ce qu'on pourrait appeler le "paradoxe de la linéarité »: le traducteur fabrique idéalement un hypertexte qu'il lui faut ensuite « réduire » à un texte linéaire, unique. La liberté du traducteur car elle existe - reste certainement de choisir parmi la gamme de possibilités qu'offre la langue d'arrivée. Le texte source peut donc être vu comme un "monde déjà à disposition » et l'opération de traduction comme ce qui élit un « monde possible » au prix de la réfutation d'autres mondes qui, à un moment donné, furent également possibles. Pour autant, lorsque subsiste la trace des mondes reniés, son examen est une étape indispensable de la constitution du dossier génétique.

Voici un exemple illustrant la façon dont un ajout et une suppression du traducteur visent la rectification d'un contresens initialement commis. L'exemple est emprunté au tapuscrit, déposé à l'IMEC, de quelques feuillets de la traduction, par Gilles Barbedette, d'une nouvelle de Vladimir Nabokov, The Enchanter ${ }^{34}$. Cette nouvelle, que l'auteur voyait comme le "prototype de Lolita », fut initialement (en 1939) écrite en russe. Le manuscrit en fut longtemps perdu, avant d'être retrouvé et traduit en anglais par Dimitri Nabokov. C'est à partir de la traduction en langue anglaise que Gilles Barbedette a établi sa version française. La transcription du tapuscrit est mienne 35 (fig. 4).

Il n'est pas utile de se référer au texte source, ni d'ailleurs à la version imprimée de la traduction pour comprendre la logique qui a présidé à la biffure, à la cinquième ligne, du segment «en avance sur son temps » et avancer l'hypothèse que l'ajout de « prématurément» (à la quatrième ligne) est postérieur à la biffure. Barbedette a ici corrigé un contresens, « en avance sur son temps » ne caractérisant pas la jeune fille, mais le détournement précoce de l'attention. Ce que le repentir fait aussi apparaître est que

34. Vladimir Nabokov, The Enchanter, traduit du russe par Dimitri Nabokov (1986), New York, Vintage International, Random House Inc., 1991 ; L'Enchanteur, traduit de l'anglais pas Gilles Barbedette, Postface de Dmitri Nabokov, Paris, Rivages poche, 2000. Le titre de la nouvelle en russe est Volshebnik.

35. N'ayant pas été autorisée à photocopier le tapuscrit, je l'ai dactylographié et ai reproduit les annotations du traducteur. 
familier mais jarnais ennuyeux. Il était convaincu qu'aussi longtemps que la nouveauté prévaudrait et qu' elle ne regarderait pas autour d'elle, il serait facile, par le biais de petits noms et de plaisanteries confinmant ateatovie la simplicitén essentiellement frile de bizarreries données, de détourner i'attention d'une fille incentrime

normale des comparaisons, des généralisations et des

questions qui pourraient être suggérées par une phrase entendue auparavant, par un rêve ou bien pa première menstruation, afin de préparer une transition sans entre un probablement douleur pur monde de semi-abstractions dont elle était ấ moitié consciente spoutanèment

(telles que l'interprétation correcte du ventre gonflé t.autonemé d'une voisine belle gueule

ou bien la prédilection d'une écolière pour la d'une idole du public entre

féminin) tout ce qui était lié d'une manière ou d'une autre à l'amour adulte de I a sumbut

et la réalité taicindis que la bienséance et la moralité, ignorant des destraction agreables

la conduite aussi bien que la galanterie, s'abstiendraient de toute visite. Relever I adresse pour le

des pont-levis pourrait être un système efficace de protection jusqu'ay moment fossé en fleurs remonterait dans la chambre avec une jeune branche

Fig. 4

la même locution - en l'occurrence ahead of time - est susceptible de traductions très différentes selon sa place dans la phrase 36 .

Le travail du traducteur présente toutefois une particularité dont il faut tenir compte, notamment lorsqu'on cherche à identifier l'étape du processus correspondant à la phase prééditoriale : la remise de la traduction à l'éditeur ne constitue quasiment jamais, de nos jours, la dernière phase du travail du traducteur. Relue par un tiers - selon les cas,

36. Le contresens initial s'explique peut-être par l'extrême longueur de la phrase, faite d'un tronc principal sur lequel viennent se greffer toutes sortes d'excroissances : « He was convinced that, as long as novelty still prevailed and she did not look around her, it would be easy, by means of pet names and jokes confirming the essentially aimless simplicity of given oddities, to divert a normal girl's attention ahead of time from the comparisons, generalizations, and questions that might be prompted by something overheard previously, or a dream, or her first menstruation, so as to prepare a painless transition from a world of semiabstractions of which she was probably sem-conscious (such as the correct interprétation of a neighbor's autonomously swelling belly, or schoolgirl prédilections for the mug of a matinee idol), from everything in any way connected with adult love, into the everyday reality of pleasant fun, while décorum and morality, aware neither of the goings-on nor the address, would refrain from visiting » (p. 54-55). Je n'ai commenté ici, faute de place, que les modifications affectant la traduction de ahead of time, mais les nombreuses autres rectifications du traducteur, dont celle d'un autre contresens portant cette fois sur confirming, semblent indiquer que Barbedette a initialement traduit la phrase d'un seul élan pour y revenir ensuite (elle était de fait incompréhensible en l'état). Était-il un traducteur du «flux »? 
correcteur, réviseur et/ou personne chargée du suivi éditorial de la traduction ${ }^{37}$-, la traduction revient ensuite vers son auteur, assortie tantôt de questions concernant des choix sémantiques ou syntaxiques qu'il lui est demandé de justifier, tantôt de propositions de modification qui lui sont faites. La phase de travail qui s'ouvre alors consiste pour le traducteur à mesurer l'écart entre la lecture «tierce », telle que la matérialisent les questions ou les suggestions de modification qui lui sont adressées, et la lecture que lui-même a faite de l'œuvre, telle que la matérialise son travail de traduction. Chaque proposition de modification exige une décision de la part du traducteur - l'accepte-t-il ou la récuse-t-il ? - qui ne peut se prendre qu'après qu'il a lui-même essayé de reconstituer le processus qui a induit cette proposition-là. La première question que suscitera donc cette étape de son travail sera : pourquoi cette modification m'est-elle proposée ? Si l'on pose la question autrement - pourquoi réfute-t-on mon choix initial ? -, l'on voit bien que dans cette phase de son travail, le traducteur se fait un peu généticien ! Le temps de latence qui sépare les deux moments - celui de la remise de la traduction puis de son « retour à l'envoyeur »- opère une double mise à distance : non seulement le regard du relecteur confronte le traducteur à ses propres choix, mais lui-même, bien souvent, reconsidère son travail d'un œil sinon neuf, du moins plus frais (c'est là l'un des effets du caractère foncièrement interminable d'une traduction ; sans le processus éditorial, on peut se demander ce qui viendrait mettre un terme au lien symbiotique qui unit le traducteur à son texte). Que la confrontation entre les deux regards - étranger du relecteur ${ }^{38}$ et réflexif du traducteur - ne se fasse pas toujours sans violence s'explique par le fait que même le relecteur le plus respectueux du travail de traduction n'a jamais la connaissance intime de l'œuvre que s'est acquise le traducteur en en ôtant patiemment les voiles, au risque de mettre à nu le roi lui-même. Ainsi cette remarque de Derrida au traducteur allemand de La Carte postale, Hans Joachim Metzger : «À lire vos questions, je vois une fois encore que vous avez mieux lu le texte que moi. C'est pourquoi un traducteur est absolument insupportable, et meilleur il est, plus il fait peur : le surmoi en personne ${ }^{39}$. »

La remarque de Derrida suggère la possibilité d'un effet rétroactif de la traduction et le dit puissant, dérangeant. L'ample question qu'elle laisse entrevoir est celle des modalités selon lesquelles la traduction signifie pour l'œuvre (et non pas seulement pour l'auteur de l'œuvre, mis face à son double surmoïque). Herder et Gœthe, au XvIII siècle, avaient bien perçu l'effet de rajeunissement (Verjüngung) qu'opère la traduction en prêtant à une œuvre la langue de son temps. Mais Derrida rehausse la question de l'effet retour en faisant du traducteur un hyperlecteur. Est-ce là inviter à penser la traduction comme geste critique, ainsi que l'avait pressenti, sans toutefois le théoriser, Ezra Pound en 1934 ? Il y a fort à parier que nous avons beaucoup à apprendre de l'éclairage que la traduction, examinée sous l'angle génétique, « rétro-projette » sur l'œuvre pour sonder sa part la plus secrète.

37. La traduction peut être aussi soumise au regard de l'auteur de l'original, voire à son approbation. 38. Le regard du réviseur opère parfois comme un « choc exogène », pour reprendre l'expression très juste de Daniel Ferrer dans Logiques du brouillon. Modèles pour une critique génétique, Paris, Éditions du Seuil, 2011, p. 55 sq. 39. Cité par Benoît Peeters, dans Derrida, Paris, Flammarion, 2010, p. 387. 
Fabienne Durand-Bogaert, agrégée d'anglais, docteur en sciences du langage, enseigne l'histoire et la critique des traductions à l'EHESS. Aux publications liées à ses activités de recherche - de nombreux articles sur la traduction, une monographie sur Peter Handke et, en collaboration avec Yves Hersant, l'anthologie Europes (Laffont, 2001) - s'ajoute la traduction d'une trentaine d'ouvrages de sciences humaines (philosophie, histoire de l'art, critique de l'art moderne et contemporain, anthropologie) parus pour la plupart aux éditions Gallimard.

fabienne.durand-bogaert@orange.fr

Résumés

Les deux corps du texte

La secondarité de la traduction par rapport à l'œuvre que l'on dit « originale » est une donnée essentielle de la configuration qui se présente au généticien. Ses conséquences - fragilisation de l'auctoritas du traducteur, marginalisation de la traduction au sein de l'espace littéraire - pèsent sur la disponibilité de l'archive et sa nature. À partir de quels documents et selon quelles procédures peut-on envisager une approche génétique des traductions, telle est la question à laquelle l'article s'efforce de répondre en illustrant sa visée théorique d'exemples concrets.

The fact that a translation always comes after the "original" work impacts greatly on genetic criticism. Indeed, by weakening the translator's auctoritas and marginalising translation within the literary field, it affects the nature of translators' manuscripts and typescripts and reduces the opportunity to access them. This article will attempt to describe, through the use of concrete examples, the kind of documents and procedures on which a genetics of translation can be founded.

Die zeitliche Nachstellung der Übersetzung gegenüber dem „originalen” Werk ist eine grundlegende Gegebenheit für die Arbeit des Genetikers. Die Schwächung der auctoritas des Übersetzers und der marginalisierte Stellenwert der Übersetzung innerhalb des literarischen Raumes haben einen schwerwiegenden Einfluss auf die Verfügbarkeit und die Art der Dokumente. Anhand einer theoretischen Betrachtung konkreter Beispiele will dieser Artikel der Frage nachgehen, welche Dokumente und welche Verfahren sich für eine genetische Untersuchung der Übersetzung anbieten.
El carácter subalterno de la traducción con respecto a la obra llamada "original" es un dato esencial de la configuración que debe tomar en cuenta el geneticista. Sus consecuencias -fragilización de la auctoritas del traductor, marginalización de la traducción en el seno del espacio literario- gravitan sobre la disponibilidad del archivo y su naturaleza. ¿Cuáles son los documentos y los procedimientos a partir de los cuales se puede establecer un enfoque genético de las traducciones? Es éste el interrogante que el presente artículo intenta responder, ilustrando sus objetivos teóricos con ejemplos concretos.

A dependência da tradução em relação à obra dita "original" é um dado essencial no quadro que se oferece ao geneticista. A reduzida"auctoritas" do tradutor ou o estatuto marginal da tradução no espaço literário têm efeito sobre a disponibilidade do arquivo e a sua natureza. Com que documentos e com que procedimentos se pode conceber uma abordagem genética das traduções - esta é a pergunta a que o artigo procura responder, ilustrando a sua visão teórica com exemplos concretos.

E un fatto che la secondarietà della traduzione in rapporto a quello che chiamiamo 1'“originale" è un elemento essenziale della configurazione che si presenta al genetista. Le conseguenze - svilimento della auctoritas del traduttore, marginalizzazione di quella traduzione nello spazio della cultura letteraria - pesano certo sulla natura e sulla utilizzabilità dei materiali d'archivio. Il saggio prova a descrivere, ricorrendo ad esempi concreti, talune tipologie di documenti e i relativi procedimenti su cui può fondarsi una genetica della traduzione. 